\title{
Understanding radio polarimetry. II. Instrumental calibration of an interferometer array
}

\author{
R.J. Sault ${ }^{1,2}$, J.P. Hamaker ${ }^{1}$ and J.D. Bregman ${ }^{1}$ \\ 1 Netherlands Foundation for Research in Astronomy, Postbus 2, 7990 AA Dwingeloo, The Netherlands \\ 2 Australia Telescope National Facility, CSIRO, P.O. Box 76, Epping, N.S.W. 2121, Australia
}

Received August 4; accepted October 22, 1995

\begin{abstract}
In a companion paper, a mathematical formalism to describe the polarimetric response of a radio interferometer was presented. Some of the instrumental parameters, however, are either unknown or poorly known. Here we consider the determination of these parameters both by a traditional radio-interferometry instrumental approach as well as by using optical polarimetry principles. In doing so, we establish links between the two fields. We show that some degrees of freedom cannot be solved for with various calibration or self-calibration schemes. These degrees of freedom are identified with instrumental parameters and physical source properties. The number of unsolvable degrees of freedom is reduced for a long synthesis with alt-az antennas. We also consider the effect of errors in the assumed instrumental parameters on the resultant calibrated data. The polarimetric calibration procedure for some telescopes is reviewed in the context of this analysis.
\end{abstract}

Key words: methods: analytical — methods: data analysis — techniques: interferometers — techniques: polarimeters

\section{Introduction}

Polarimetric properties of an astronomical source provide important extra probes on the physical processes present. Given the technical simplicity of making polarimetric measurements at radio frequencies, many radio telescopes produce polarimetric images as a matter of course. However, polarimetric calibration of the instrument remains important. In the case of interferometer arrays, Morris et al. (1964) were the first to address calibration issues. Although various papers have appeared in the literature on the polarimetric calibration of interferometers (e.g. Weiler 1973; Bignell 1982), they have concentrated on specific instruments and receptor configurations. Their approach has been based on instrumental parameters. This, in turn, has resulted in a lack of generality - the limits of the polarimetric calibration of radio interferometers in general have not been properly addressed.

In a companion paper (Hamaker et al. 1996, hereafter Paper I), we describe an approach to describing the polarimetric response of an interferometer. This approach is a generalization of a traditional optical approach, and so helps unite optical and radio-interferometric polarimetry. We consider the problem of polarimetrically calibrating an interferometer array and show that results from optical polarimetry can also be applied in this problem. We show

Send offprint requests to: J.D. Bregman that analogues with optical polarimetry help place limits on the number of instrumental parameters that cannot be determined by various calibration and self-calibration approaches. In particular, three degrees of freedom cannot be determined from a single calibrator observation, and seven degrees of freedom cannot be determined in self-calibration. We identify these degrees of freedom both with some instrumental parameters and physical properties. For alt-az antennas (for long observations), the number of unsolvable degrees of freedom is reduced.

In the following, we consider an interferometric array composed of $N$ antennas. We will assume that each antenna is equipped with two receptors which are sensitive to nominally opposite polarizations (e.g. two circularly or two linearly polarized receptors). We will also assume that there are at least three antennas and that all four possible correlations between all antenna pairs are measured simultaneously. We stress that Paper I generally considered a single baseline only. Some of our results do not apply to calibrating a single baseline in isolation or when all possible baselines and correlations are not measured - closure of the array is necessary. We will not consider off-axis polarimetric effects (strictly we consider the polarimetric response at just one part of the primary beam), nor will we consider frequency variation of the response.

At times we will consider specific receptor configurations. The two most commonly found in radio 
interferometry are the so-called 'circular' and 'parallellinear' configurations. These are arrays with circularly or linearly polarized receptors. For simplicity we assume that all receptors have the same alignment. More so, except when considering alt-az antennas, we assume that this alignment is along the vertical (in the convention of $\mathrm{Pa}$ per I, we assume no rotation). We also sometimes consider the 'crossed-linear' configuration, which is an array of linearly polarized receptors where at least one receptor pair is rotated by $45^{\circ}$ relative to the others (Weiler 1973).

\section{Summary and notation of Paper I}

Summarizing the results and notation of Paper I, for a given baseline, the measured visibilities can be described by a 4 -element vector,

$$
\boldsymbol{v}=\left(\begin{array}{l}
v_{\mathrm{pp}} \\
v_{\mathrm{pq}} \\
v_{\mathrm{qp}} \\
v_{\mathrm{qq}}
\end{array}\right) .
$$

Here subscripts $\mathrm{p}$ and $\mathrm{q}$ represent the two polarization channels measured by each antenna. The vector is best described as an instrument-based coherency vector.

This coherency vector is related to the so-called source 'Stokes visibility vector' ${ }^{1}, s=(I, Q, U, V)^{\mathrm{T}}$ by a matrix operation,

$$
\boldsymbol{v}=\boldsymbol{K s}
$$

Here $\boldsymbol{K}$ is a $4 \times 4$ matrix. For the purposes of this paper, it is convenient to replace $\boldsymbol{K}$ by its factors,

$$
\boldsymbol{v}=\left(\boldsymbol{J}_{i} \otimes \boldsymbol{J}_{j}^{*}\right) \boldsymbol{S} \boldsymbol{s}
$$

Here $\boldsymbol{S}$ is a $4 \times 4$ matrix which converts a Stokes vector into a coherency vector (ignoring instrumental effects). As with Paper I, where we need to specify a convention for the output of $\boldsymbol{S}$, we use an ' $x y$ ' representation in the frame of the sky. This in no way limits the discussion to a particular feed type. It is simply a convenient coordinate system. The $2 \times 2$ matrices $\boldsymbol{J}_{i}$ and $\boldsymbol{J}_{j}$ are antenna-based response matrices, which are known as Jones matrices in optical polarimetry (the subscripts $i$ and $j$ represent the antenna numbers). The operator $\otimes$ represents the matrix outer product (also known as the direct, tensor or Knonecker product; see Paper I). In many ways the antenna Jones matrix can be thought of as the polarimetric generalization of the simple antenna gain of non-polarimetric interferometry.

\footnotetext{
${ }^{1}$ As Paper I stresses, in the sense that a vector is a representation of a physical quantity independent of a coordinate system, the Stokes and coherency parameters are two alternative representations of the one physical vector. Paper I's terminology was equally strict. Here we abandon this in favour of convenience - we use the terms coherency vector and Stokes vector.
}

The antenna Jones matrix can be further decomposed into matrices which model various aspects of the signal path, viz

$$
\boldsymbol{J}_{i}=\boldsymbol{G}_{i} \boldsymbol{D}_{i} \boldsymbol{C}_{i} \boldsymbol{P}_{i} \boldsymbol{F}_{i}
$$

Here $\boldsymbol{G}_{i}, \boldsymbol{D}_{i}, \boldsymbol{C}_{i}, \boldsymbol{P}_{i}$ and $\boldsymbol{F}_{i}$ represent $2 \times 2$ antenna-based gain, feed (which models the leakage process), nominal feed configuration, parallactic angle rotation and Faraday rotation matrices respectively. Paper I gives their form.

We will be particularly interested in the matrices modelling the gains and leakages,

$$
\boldsymbol{G}_{i}=\left(\begin{array}{cc}
g_{i \mathrm{p}} & 0 \\
0 & g_{i \mathrm{q}}
\end{array}\right)
$$

and

$$
\boldsymbol{D}_{i}=\left(\begin{array}{cc}
1 & d_{i \mathrm{p}} \\
-d_{i \mathrm{q}} & 1
\end{array}\right)
$$

The gain and leakage coefficients are complex valued ('gain' will always imply a complex-valued gain - gain amplitude and gain phase). We stress that, contrary to the suggestion in some references, modelling feed errors by leakages does not involve any small-error approximation. The leakage model is based on the assumed linearity of the response of the receptor to emission, and models large receptor errors equally well - see Paper I for the argument.

\section{Analogues with optical polarimetry}

Here we demonstrate some connections of the calibration problem with optical polarimetry (see Paper I; Azzam \& Bashara 1987, pages 148-158, or many texts on optical polarimetery for background which is helpful with this section). We consider a radio interferometer array, its correlator and imaging hardware and software as a single polarimetric optical system; given a source in the sky, polarimetric images are formed on our computer displays. Linearity of the system is the key property here (non-linear deconvolution, for example, is forbidden - undeconvolved images are adequate for the arguments we will make). The optical results we will use in this section rely only on the linearity of the optical system. For simplicity, we will consider the problem of estimating the Stokes parameters of a point source at the phase centre. These estimates will simply be the sum of the Stokes visibilities (whether these visibilities have had calibration applied is not relevant at this stage). Because of the linearity of the overall process, the relationship between the true Stokes parameters and the estimates can be represented by a matrix. In optics, this $4 \times 4$ matrix is known as the Mueller matrix, $\boldsymbol{M}$; it describes the polarimetric response of a system.

Note that the Mueller matrix describes the polarimetric response only. A full characterization of an instrument would include a point-spread function and (for a spectral instrument) a spectral response function. Although they 
could be included in the treatment, they are not important to the argument.

To determine the Mueller matrix of an interferometer array, consider the effective Jones matrix of each antenna of the array after calibration. In non-polarimetric radiointerferometry, the calibration solution step consists of estimating the antenna gains, and then correcting the data for these gains. Likewise in polarimetric interferometry, calibration can be thought of as estimating the antenna Jones matrix, and then correcting the data for this Jones matrix. The effective Jones matrix of an antenna after calibration is then

$$
\boldsymbol{J}_{i, \text { eff }}=\boldsymbol{J}_{i, \text { est }}^{-1} \boldsymbol{J}_{i, \text { true }}
$$

Ideally, our estimate will be completely correct, and the effective Jones matrix of the antenna will be the identity matrix.

Assuming equal weights for all visibilities (i.e. natural weighting), the Mueller matrix for a nominally calibrated system is then (for a point source at the phase centre)

$$
\boldsymbol{M}=\frac{1}{N(N-1)} \sum_{i}^{N} \sum_{j, j \neq i}^{N} \boldsymbol{S}^{-1}\left(\boldsymbol{J}_{i, \mathrm{eff}} \otimes \boldsymbol{J}_{j, \mathrm{eff}}^{*}\right) \boldsymbol{S}
$$

(note that, to get a real output, this sums over both a baseline and its conjugate baseline).

One of the results of optical polarimetry is that a Mueller matrix which describes a pure system (a system which does not depolarize fully polarized emission - see Paper I for a fuller discussion) can be equivalently expressed in terms of a single Jones matrix, $\boldsymbol{J}$ :

$$
\boldsymbol{M}=\boldsymbol{S}^{-1}\left(\boldsymbol{J} \otimes \boldsymbol{J}^{*}\right) \boldsymbol{S} .
$$

A system which is not pure will partially depolarize some fully polarized emission. Obviously a system that does not change the polarization state of the emission is pure ( $\boldsymbol{J}$ and $\boldsymbol{M}$ are the identity matrices). However to say a system is 'pure' does not mean that the output has the same polarization as the input - the polarization state can be changed. For example a system which rotates the position angle of linear polarization is pure.

Note that, as the Jones matrix $\boldsymbol{J}$ contains four complex coefficients, and as the phase of one of these is arbitrary, the Mueller matrix of a pure system has only seven degrees of freedom - the 16 numbers in a pure Mueller matrix are not independent. This is not saying that the internal state of the optical system is describable by just seven numbers, but that its overall polarimetric response is.

Under what circumstances is our interferometric imaging system pure? If it is perfectly calibrated, then clearly it is pure. Indeed, even if it is not perfectly calibrated, but if the effective Jones matrices of all antennas are the same, then the system will be pure. In general, however, an uncalibrated interferometer array is not a pure system. It is the calibration process which makes it pure.
Consider trying to calibrate an interferometer array from an observation of a point source, at the phase centre, whose polarization and flux density is completely unknown. Because the source's polarimetric characteristics are unknown, any set of instrumental parameters which is consistent with a pure system is a feasible solution. That there are other consistent pure solutions is easy to show. If all the estimates for antenna Jones matrices are in error by a constant Jones matrix,

$$
\boldsymbol{J}_{i, \text { true }}=\boldsymbol{J}_{i, \text { est }} \boldsymbol{J}_{\text {err }},
$$

then, after calibration is applied, the effective Jones matrix of all antennas is constant:

$$
\boldsymbol{J}_{i, \mathrm{eff}}=\boldsymbol{J}_{\mathrm{err}},
$$

and then Eq. (8) shows the Mueller matrix of the system to be

$$
\boldsymbol{M}=\boldsymbol{S}^{-1}\left(\boldsymbol{J}_{\text {err }} \otimes \boldsymbol{J}_{\text {err }}^{*}\right) \boldsymbol{S} .
$$

The fact that there are many consistent solutions has an equivalent in non-polarimetric calibration. Consider calibrating an interferometer array with an observation of a point source of unknown flux density. In this case we cannot determine the absolute flux gain. In the nonpolarimetric case, reflecting that we do not know the calibrator flux density, the antenna gain can be multiplied by an arbitrary gain factor and a consistent solution is retained. In the polarimetric case, reflecting that we do not know the calibrator flux density or polarization, the antenna Jones matrix can be multiplied by an arbitrary Jones matrix and a consistent solution is retained.

There is a significant difference between the nonpolarimetric and polarimetric cases in terms of degrees of freedom. In the non-polarimetric case, there is one degree of freedom unspecified. In the polarimetric case, the error Jones matrix has seven degrees of freedom. This means that, with a calibrator of unknown polarization, at least seven (possibly more) degrees of freedom cannot be determined in the solution process. There is also a significant difference in the astrophysical importance of these degrees of freedom. A small error in the absolute flux may not be important, whereas corruption of $V$ by $I$ may be of paramount importance.

What is possible if the polarization of the source is known? If the source's polarization is known, then four extra degrees of freedom (corresponding to the four Stokes parameters of the source) are constrained in the solution process. This does not provide enough information to constrain the (at least) seven degrees of freedom. Thus we see that a single observation of a point-source calibrator cannot determine at least three degrees of freedom.

\section{Solving with an unpolarized calibrator}

In this section we will show that, from an observation of an unpolarized calibrator, the instrumental parameters 
can be solved to within three degrees of freedom. That is, the limit derived by the optical analogue argument is achieved.

We consider an unpolarized calibrator, not so much because calibrators are unpolarized, but because most calibrators are only weakly polarized (typically a few percent linearly polarized and much less than one percent circularly polarized), and the polarized emission is insufficiently strong to be useful for calibration purposes.

Initially our approach will be intuitive rather than formal, with only a sketch given of the proof for a partially linearized system for parallel-linear or circular configurations. In this we assume a near-ideal instrument with gains near 1 , and small leakages. We will use $\Delta g$ as the deviation of the gains from 1 (if the gains were not normalized to 1 , we would have defined $\Delta g$ as the fractional deviation of a gain from its nominal value).

Using the formalism of Paper I, we arrive at what might be called the 'basic equations' giving the response of an interferometer. For the parallel-linear configuration

$$
\begin{aligned}
& v_{\mathrm{pp}}=g_{i \mathrm{p}} g_{j \mathrm{p}}^{*}(I+Q) \\
& v_{\mathrm{pq}}=g_{i \mathrm{p}} g_{j \mathrm{q}}^{*}\left(\left(d_{i \mathrm{p}}-d_{j \mathrm{q}}^{*}\right) I+U+i V\right) \\
& v_{\mathrm{qp}}=g_{i \mathrm{q}} g_{j \mathrm{p}}^{*}\left(\left(d_{j \mathrm{p}}^{*}-d_{i \mathrm{q}}\right) I+U-i V\right) \\
& v_{\mathrm{qq}}=g_{i \mathrm{q}} g_{j \mathrm{q}}^{*}(I-Q),
\end{aligned}
$$

and for a circular configuration

$$
\begin{aligned}
& v_{\mathrm{pp}}=g_{i \mathrm{p}} g_{j \mathrm{p}}^{*}(I+V) \\
& v_{\mathrm{pq}}=g_{i \mathrm{p}} g_{j \mathrm{q}}^{*}\left(\left(d_{i \mathrm{p}}-d_{j \mathrm{q}}^{*}\right) I+Q+i U\right) \\
& v_{\mathrm{qp}}=g_{i \mathrm{q}} g_{j \mathrm{p}}^{*}\left(\left(d_{j \mathrm{p}}^{*}-d_{i \mathrm{q}}\right) I+Q-i U\right) \\
& v_{\mathrm{qq}}=g_{i \mathrm{q}} g_{j \mathrm{q}}^{*}(I-V) .
\end{aligned}
$$

Here we have ignored products of $Q, U$ and $V$ with leakages and non-linear terms in the leakages. Note that, apart from a permutation of the Stokes parameters, the two sets of equations are identical.

We note in these basic equations that the leakages only appear in pairs such as $d_{i \mathrm{p}}-d_{j \mathrm{q}}^{*}$. Consequently the equality of the basic equations is not affected if, to all antennas, an arbitrary offset is added to $d_{\mathrm{p}}$ and the conjugate of the offset is added to $d_{\mathrm{q}}$ :

$$
\begin{aligned}
& d_{\mathrm{p}}^{\prime}=d_{\mathrm{p}}+\beta \\
& d_{\mathrm{q}}^{\prime}=d_{\mathrm{q}}+\beta^{*} .
\end{aligned}
$$

That is, the equations cannot be used to solve for the absolute level of the leakages - two of the degrees of freedom that cannot be determined are a complex-valued offset to the leakages. For the parallel-linear configuration this has a simple physical interpretation for the real part: as the real part of the leakage roughly corresponds to a receptor alignment error, an observation of an unpolarized calibrator cannot determine the absolute alignment between the rotation frames of the antennas and the sky - an entirely reasonable result.
The third degree of freedom that is unsolvable is the phase relationship between the $\mathrm{p}$ and $\mathrm{q}$ gains - a single phase difference, $\psi$, cannot be determined between the two sets of channels. That this phase cannot be determined is clear if the leakages are assumed to be zero and we consider an unpolarized source. In this case, the gains in the equations for the $\mathrm{p}$ and $\mathrm{q}$ channels are totally decoupled. Although absolute phase has little meaning in interferometry, there is a relative phase relationship between the $\mathrm{p}$ and q channels, which an observation of an unpolarized calibrator does not provide. For circular configurations, this inability to determine the phase relationship also has a simple physical interpretation: for circular configurations, a rotation of the frame of the antenna with respect to the sky results only in a change in the p-q phase difference. Again the inability to measure the phase relationship with an unpolarized calibrator reflects the inability to set the absolute alignment between the rotation frame of the antennas and the sky.

If this undetermined phase is arbitrarily equally shared by the $\mathrm{p}$ and $\mathrm{q}$ channels, then equality in the basic equations is not affected by the change:

$$
\begin{aligned}
& g_{\mathrm{p}}^{\prime}=g_{\mathrm{p}} \exp (+i \psi / 2) \\
& g_{\mathrm{q}}^{\prime}=g_{\mathrm{q}} \exp (-i \psi / 2)
\end{aligned}
$$

We will find the 'small error' versions of these two equations useful in later analysis. Assuming near-ideal antennas, these are

$$
\begin{aligned}
\Delta g_{\mathrm{p}}^{\prime} & =\Delta g_{\mathrm{p}}+\frac{i}{2} \psi \\
\Delta g_{\mathrm{q}}^{\prime} & =\Delta g_{\mathrm{q}}-\frac{i}{2} \psi .
\end{aligned}
$$

Calibration practice and our numerical analysis confirm that there are three (and only three) degrees of freedom that are not solvable. Note that this assumes at least three antennas, and that all possible baselines and correlations are measured (the practical case, where a few baselines or correlations may be missing will also usually have only three unsolvable degrees of freedom). In cases where these requirements are not satisfied, there may be more than three unsolvable degrees of freedom. For example, trying to calibrate a single baseline in isolation will result in more than three unsolvable degrees of freedom. Triangles of baselines are required to provide cross-coupling in the equations to aid solution.

The above instrumental sketch shows only that three degrees of freedom are not solvable in a simple linearized case, for two particular configurations. One must always be aware of the shortcomings of linearization, particularly when terms cancel out, etc. This, however, is not a problem in this instance. A fuller analysis shows that, for arbitrary receptors, the three unsolvable degrees of freedom 
can be expressed as an error Jones matrix (as in Eq. (10)):

$$
\boldsymbol{J}_{\mathrm{err}}=\left(\begin{array}{rr}
\cos \theta & \sin \theta \\
-\sin \theta & \cos \theta
\end{array}\right)\left(\begin{array}{cc}
\cos \phi & i \sin \phi \\
i \sin \phi & \cos \phi
\end{array}\right)\left(\begin{array}{cc}
e^{i \zeta} & 0 \\
0 & e^{-i \zeta}
\end{array}\right)
$$

This is easily verified by computing the Mueller matrix $\boldsymbol{S}^{-1}\left(\boldsymbol{J}_{\text {err }} \otimes \boldsymbol{J}_{\text {err }}^{*}\right) \boldsymbol{S}$, and noting that the response to an unpolarized source is independent of $\theta, \phi$ and $\zeta$. Intuitively, we can interpret $\theta, \phi$ and $\zeta$ as absolute alignment and ellipticity errors and an unknown phase offset.

To interpret $\boldsymbol{J}_{\text {err }}$ in terms of instrumental parameters of an antenna, we have to refer the error to the appropriate place in the signal path: we effectively have to perform a coordinate transformation. Ignoring parallactic and Faraday rotation, the equivalent error as seen from the feed of the $i$ th antenna is

$$
\boldsymbol{C}_{i} \boldsymbol{J}_{\mathrm{err}} \boldsymbol{C}_{i}^{-1}
$$

(recall $\boldsymbol{C}_{i}$ is the nominal feed configuration matrix for the $i$ th antenna). For the parallel-linear case, where the nominal feed configuration matrix is the identity matrix, referring $\boldsymbol{J}_{\text {err }}$ to the feed has no effect $-\theta, \phi$ and $\zeta$ can still be interpreted as alignment, ellipticity and phase errors. Indeed the unsolvables in the previous linearized sketch can be seen as approximations of $\boldsymbol{J}_{\text {err }}$ (e.g. $\beta$ corresponds to $\sin \theta+i \sin \phi$ and $\psi / 2$ to $\zeta$ ). For a circular configuration, referring $\boldsymbol{J}_{\text {err }}$ to the feed gives the well-known result that an absolute alignment error (rotation error) manifests itself as an instrumental phase error between the different polarization channels.

Despite there being three unsolvable degrees of freedom, this does not mean that useful calibration corrections cannot be obtained. In many cases, solving for instrumental parameters is just finding the small deviations from the nominal values, and so 'reasonable' constraints can be added to confine the solution. For example, as the leakages are small, and probably randomly distributed, Brouw (1994) advocates the constraint that the average value of the leakages is 0 , e.g.

$$
\sum_{i}^{N} d_{i \mathrm{p}}+d_{i \mathrm{q}}^{*}=0
$$

This confines $\beta$ - two of the degrees of freedom. Unfortunately, for parallel-linear and circular configurations, there is no good arbitrary assumption that allows the phase difference $\psi$ to be constrained - it must be measured in some way. Appendix A considers some approaches using unpolarized sources.

It is worth noting that, although all receptor configurations must suffer from three unsolvable degrees of freedom, not all configurations are the same - the manifestation of the degrees of freedom changes with receptor type. For example, with the crossed-linear configuration (Weiler 1973), the three degrees of freedom are perhaps best described as an alignment-error offset, an ellipticity offset for receptors with no rotation, and an ellipticity offset for the receptors which are rotated by $45^{\circ}$. The phase difference parameter can be determined. Thus for the crossed-linear configuration, the three unsolvable parameters can be confined to parameters (leakages in this case) where good assumptions can be made. In this way, Weiler was able to determine useful calibration corrections with simply an unpolarized calibrator.

\section{Effects of errors in the solutions}

The aim of the calibration process as a whole is generally not to determine instrumental parameters of the antennas, but to form corrected data. To this end, the interest in instrumental parameters goes only as far as is needed to correct data. Does adding 'reasonable constraints' to the solution process produce astrophysically adequate results? What errors are introduced into the final result by these unsolvable degrees of freedom? Small-error analysis is the most effective tool for this. To this end, we will consider the effect of the three unsolvable degrees of freedom discussed in the previous section on the calibrated data. In addition, we will also consider errors in the four degrees of freedom used in the calibration process - the assumed calibrator Stokes parameters.

Let us assume a near-ideal instrument where a weakly polarized point source is used to determine the instrumental parameters. If the calibrator was assumed unpolarized during the calibration solution process, there will be an error in the resultant estimates of the instrumental parameters. Using small-error approximations and the basic equations, we find that the estimated gains and leakages are offset from their true values. For a parallel-linear configuration,

$$
\begin{aligned}
\Delta g_{\mathrm{p}}^{\prime} & =\Delta g_{\mathrm{p}}+\frac{Q}{2 I}, \\
\Delta g_{\mathrm{q}}^{\prime} & =\Delta g_{\mathrm{q}}-\frac{Q}{2 I}, \\
d_{\mathrm{p}}^{\prime} & =d_{\mathrm{p}}+\frac{U+i V}{2 I}, \\
d_{\mathrm{q}}^{\prime} & =d_{\mathrm{q}}-\frac{U-i V}{2 I} .
\end{aligned}
$$

The primed quantities are the instrumental parameters estimated by the calibration solution process, whereas the unprimed quantities are the true values. Apart from exchanging $Q, U$ and $V$ with $V, Q$ and $U$ respectively, the results for the circular configuration are identical. We also consider another possible error in the calibrator characteristics - the assumed flux density of the calibrator may be incorrect. Such an error will result in the determined gains being offset according to the fractional error in the flux density:

$$
\Delta g_{\mathrm{p}}^{\prime}=\Delta g_{\mathrm{p}}+\frac{\Delta I}{2 I}
$$




$$
\Delta g_{\mathrm{q}}^{\prime}=\Delta g_{\mathrm{q}}+\frac{\Delta I}{2 I} .
$$

To show how errors in the instrumental parameter estimates affect the data after calibration is applied, we follow Kesteven's (1984) approach, and define

$$
\begin{aligned}
& \gamma_{++}=\left(\Delta g_{i \mathrm{p}}+\Delta g_{i \mathrm{q}}\right)+\left(\Delta g_{j \mathrm{p}}^{*}+\Delta g_{j \mathrm{q}}^{*}\right) \\
& \gamma_{+-}=\left(\Delta g_{i \mathrm{p}}-\Delta g_{i \mathrm{q}}\right)+\left(\Delta g_{j \mathrm{p}}^{*}-\Delta g_{j \mathrm{q}}^{*}\right) \\
& \gamma_{--}=\left(\Delta g_{i \mathrm{p}}-\Delta g_{i \mathrm{q}}\right)-\left(\Delta g_{j \mathrm{p}}^{*}-\Delta g_{j \mathrm{q}}^{*}\right) \\
& \delta_{++}=\left(d_{i \mathrm{p}}+d_{i \mathrm{q}}\right)+\left(d_{j \mathrm{p}}^{*}+d_{j \mathrm{q}}^{*}\right) \\
& \delta_{+-}=\left(d_{i \mathrm{p}}-d_{i \mathrm{q}}\right)+\left(d_{j \mathrm{p}}^{*}-d_{j \mathrm{q}}^{*}\right) \\
& \delta_{-+}=\left(d_{i \mathrm{p}}+d_{i \mathrm{q}}\right)-\left(d_{j \mathrm{p}}^{*}+d_{j \mathrm{q}}^{*}\right) \\
& \delta_{--}=\left(d_{i \mathrm{p}}-d_{i \mathrm{q}}\right)-\left(d_{j \mathrm{p}}^{*}-d_{j \mathrm{q}}^{*}\right) .
\end{aligned}
$$

Note that there are seven parameters (there is no $\gamma_{-+}$). Using the small-error approximations detailed in Appendix B, the errors in the deduced Stokes visibilities, $\boldsymbol{\Delta} \boldsymbol{s}$, for a parallel-linear configuration, are

$$
\boldsymbol{\Delta} \boldsymbol{s}=-\frac{1}{2}\left(\begin{array}{rrrr}
\gamma_{++} & \gamma_{+-} & \delta_{+-} & -i \delta_{-+} \\
\gamma_{+-} & \gamma_{++} & \delta_{++} & -i \delta_{--} \\
\delta_{+-} & -\delta_{++} & \gamma_{++} & i \gamma_{--} \\
-i \delta_{-+} & i \delta_{--} & -i \gamma_{--} & \gamma_{++}
\end{array}\right) \boldsymbol{s}
$$

The circular case is the same except that there is a permutation to interchange $Q, U$ and $V$ in the above with $V$, $Q$ and $U$ respectively.

Each of the seven $\gamma$ and $\delta$ parameters are potentially affected by an error in the assumed calibrator polarization or by one of the unsolvable degrees of freedom. That is, there are seven error mechanisms. These are summarised in Table 1. Of these seven error mechanisms, only either the real part or the imaginary part of each coefficient contributes to the error. That is, there are seven degrees of freedom in these error mechanisms. That there are seven the same number of degrees of freedom in a pure Mueller matrix - is not by chance. This merely reflects that there are seven 'self-consistent' ways for a pure polarimetric system to be in error.

It is interesting, and intuitively reasonable, to note that an error in the assumed calibrator polarization results in a spurious polarized source of the same type and percentage but of opposite sign in the data after calibration. Indeed these are the only causes of $I$ corrupting $Q$, $U$ and $V$. Note also that the degrees of freedom that are not solvable with an unpolarized calibrator do not cause $I$ to corrupt $Q, U$ or $V$ (again intuitively correct).

It should be stressed that these seven error mechanisms result in self-consistent images. Although the images are in error, the error is not apparent. The dynamic range of the images is not affected by the errors. This is not a consequence of the small-error analysis. The errors may or may not be of astrophysical significance. For example, a misalignment of $1^{\circ}$ in the position angle of linear polarization is possibly not important, whereas a small corruption of $V$ by $I$ is possibly of paramount importance.

\section{Solving with polarized calibrators}

The previous sections showed that a single observation of a point-source calibrator could not, at best, solve for three degrees of freedom in the instrumental parameters. Furthermore, this limit was achieved with an unpolarized calibrator. Not unreasonably, this can also be shown to be true for an arbitrarily polarized calibrator. Interestingly, although one observation leaves three degrees of freedom unsolvable, if the calibrator has significant linear polarization, three 'reasonable' constraints on the leakages can be used to allow 'reasonable' estimates of the instrumental parameters (including $\psi$ ) to be determined. For example, using Eq. (29) and

$$
\sum_{i}^{N} d_{i \mathrm{p}}+d_{i \mathrm{q}}=0
$$

are sufficient constraints for the purpose (note that these two complex-valued equations constrain only three degrees of freedom - the constraints on the real parts of the two equations are the same). Combining these constraints with a polarized calibrator fixes $\psi$ and $\beta$.

If we do not choose to make such assumptions, a natural question to ask is what observations are needed to completely determine the instrumental parameters. This is the same as asking what observations are needed to determine the parameters of a pure optical system. From the optical literature, we find the counter-intuitive result that three 'distinct' observations are needed (Azzam \& Bashara 1987). This is somewhat counter-intuitive because simplistic arguments suggest that two observations (with four Stokes parameters specified per observation) will potentially uncover the seven degrees of freedom in a pure Mueller matrix. Unfortunately the structure of this form of Mueller matrix makes this simplistic argument false.

An alternative way of determining this result is to consider the $\delta$ and $\gamma$ matrix of Eq. (43). Let us expand the matrix into linear equations, with the Stokes parameters as the coefficients and the $\delta$ and $\gamma$ parameters as the unknowns. How many separate sets of Stokes parameters are needed to solve for the $\delta$ and $\gamma$ parameters? A small amount of analysis shows that three sets of Stokes parameters are required to make this system of linear equations non-singular. Furthermore it will be non-singular if, and only if, the three Stokes vectors are linearly independent.

What types of observations suffice? Clearly no amount of re-observing unpolarized sources (whether point sources or extended) will help; they only provide one 'distinct' observation. At least two of the observations must be polarized with at least one of these being linearly polarized (a circularly polarized calibrator is not essential). Interestingly, a long observation of a linearly polarized source with alt-az antennas (i.e. significant parallactic angle rotation - see Sect. 7) suffices for the three distinct observations. 
Table 1. Summary of corrupting influences in polarimetric calibration. Each of the seven $\gamma$ and $\delta$ terms are potentially affected by a wrong assumption of the calibrator or a poorly determined parameter in the calibration process. This causes a corrupting influence. Most commonly this results in one Stokes parameter corrupting another, and vice versa. We symbolise this with $\Leftrightarrow$. For example, $I \Leftrightarrow Q$ means "I corrupts $Q$, and $Q$ corrupts $I$ "

\begin{tabular}{|c|c|c|c|c|}
\hline Error term & Corrupting cause & $\begin{array}{l}\text { Relevant } \\
\text { equations }\end{array}$ & $\begin{array}{l}\text { Effect on parallel-linear } \\
\text { configuration }\end{array}$ & $\begin{array}{l}\text { Effect on circular } \\
\text { configuration }\end{array}$ \\
\hline$\gamma_{++}$ & Wrong calibrator flux & 34,35 & Incorrect flux scale & Incorrect flux scale \\
\hline$\gamma_{+-}$ & Wrong calibrator $Q$ or $V$ & 30,31 & $I \Leftrightarrow Q$ corruption & $I \Leftrightarrow V$ corruption \\
\hline$\gamma_{--}$ & Poor $\psi$ & 25,26 & $U \Leftrightarrow V$ corruption & Error in angle of linear pol'n \\
\hline$\delta_{++}$ & Real leakage offset & 21,22 & Error in angle of linear pol'n & $Q \Leftrightarrow V$ corruption \\
\hline$\delta_{+-}$ & Wrong calibrator $U$ or $Q$ & 32,33 & $I \Leftrightarrow U$ corruption & $I \Leftrightarrow Q$ corruption \\
\hline$\delta_{-+}$ & Wrong calibrator $V$ or $U$ & 32,33 & $I \Leftrightarrow V$ corruption & $I \Leftrightarrow U$ corruption \\
\hline$\delta_{--}$ & Imaginary leakage offset & 21,22 & $Q \Leftrightarrow V$ corruption & $U \Leftrightarrow V$ corruption \\
\hline
\end{tabular}

Additionally, a single observation of an extended source (at least three resolution cells in size), with varying (nonzero) fractional polarization, may also suffice.

\section{Rotation}

So far, for simplicity, we have assumed receptors with no rotation. We have not lost any generality in doing so. Including rotation of the receptors from the vertical merely causes $Q$ and $U$ to be replaced with a rotated $Q$ and $U$. Paper I shows that this is straightforward to include.

However, rotating a receptor relative to the sky, such as the parallactic angle rotation of a feed on an alt-az mount, can be used to good advantage when solving for instrumental parameters that are constant with time. This is because the instrumental parameters are in the frame of the antennas, whereas the source parameters are in the frame of the sky. By allowing rotation between the two, and assuming good parallactic angle coverage, instrumental parameters (within limits) and calibrator linear polarization can be solved for simultaneously (see Conway \& Kronberg 1969). As many calibrators will often have small linearly polarized components, which vary with timescales of months, it is often best to treat the linear polarization of a calibrator as an unknown.

Physically rotating the receptors can also be used as a calibration technique. However, as Weiler \& Wilson (1977) note, the act of rotating the receptors does affect the polarimetric response, and so should be used with caution.

For a calibrator with significant linear polarization, with sufficient parallactic angle coverage this technique can be used to solve for two of the degrees of freedom that cannot be determined with an unpolarized calibrator (appropriate analysis of multiplying the matrix of Eq. (43) by a rotation Mueller matrix will show that $\delta_{--}$and $\gamma_{--}$ can be determined). The one remaining 'unsolved' degree of freedom is, very reasonably, the absolute alignment be- tween the antenna and sky rotation frames (corresponding to $\delta_{++}$in Eq. (43)).

No equivalent technique exists to determine $V$. That $V$ is insensitive to rotation follows immediately from its physical definition (the methods of Paper I, or most other interferometer response equations, show this formally).

Apart from parallactic rotation, the Faraday effect in the ionosphere may also introduce a significant apparent rotation between the sky and an antenna. However, unlike parallactic angle rotation, it is not known and so is not a tool for, but rather a hindrance to, the calibration process.

As Faraday rotation is not a part of the interferometer hardware, and as we have shown that interferometer errors could be identified as the three degrees of freedom that cannot be determined from a single observation of a calibrator, it is apparent that the effects of Faraday rotation cannot be disentangled from interferometer errors. That is, Faraday rotation effects can be subsumed into the gain and leakage matrices. This is somewhat like antenna phase, where we cannot distinguish between the atmospheric and instrumental components.

For the circular configuration, Faraday rotation can be subsumed into the phase difference $\psi$, whereas for the linear configuration it can be subsumed into the real part of the leakages (at least in a small-error sense - for the leakage model used here, large Faraday rotations will affect both the leakages and gains). In general, if all the 'instrumental parameters' are solved for astronomically (from a source above the ionosphere), then the ionospheric Faraday rotation component will have been accounted for. In this case, however, it is not appropriate to assume that the leakages are small (linear configuration) or that a noise-injection system provides a good estimate of $\psi$ (circular configuration). As Faraday rotation typically varies during the course of a long synthesis, regular observations of a linearly polarized calibrator would be needed to track the changes. 
Faraday rotation can often be estimated by other methods - see Thompson et al. (1986) for a summary.

\section{Practical calibration strategies}

The preceding sections have concentrated on limits to determining the instrumental parameters. Here we review the more practical issue of actual solution strategies.

In the solution process, the leakage and gain coefficients form coupled non-linear equations. They contain products between leakages, products between gains and products between these leakages and gains. For an $N$ antenna array, as the gain and feed matrices are antennabased, we will have only of the order of $N$ unknown matrices, but we have measurements of $N(N-1) / 2$ baselines. That is, as with normal non-polarimetric "antenna gain" calibration, there will be more measured quantities than unknowns. Consequently the appropriate way to solve for the parameters is by a least-squares approach (or similar). In principle, the full, coupled, non-linear equations should be solved. With appropriate algorithms, the computational penalty for such an approach is often insignificant. However, most current packages use various approximations or assumptions to linearize or partially linearize the equations, or to decouple the gain and leakage solution process.

In the solution process, often some of the instrumental parameters can be assumed to remain constant over the observation, whereas others can not. For example, leakages (mostly mechanical in origin) are generally stable over the length of an observation, whereas gains (electronic and atmospheric in origin) vary more rapidly. With good electronic design, it may be reasonable to assume that the phase difference $\psi$ is also constant over an observation. If Faraday rotation is significant and time-variable, then the manner in which it is treated will affect whether leakages (for linear configurations) or $\psi$ (for circular configuration) can be assumed constant over an observation.

Whereas the polarization calibration for the parallellinear and circular configurations show great mathematical similarity, there are significant practical differences between the two. This arises because it is usually reasonable to assume that a calibrator will have negligible circular polarization $(V<0.2 \%)$, whereas the fractional linear polarization will typically be a few percent (e.g. Weiler \& de Pater 1983).

For the circular configuration, assuming that the calibrator is not strongly linearly polarized $(<10 \%)$ and/or that the leakages are small $(<3 \%)$, the gain (excluding $\psi$ ) and leakage solutions are separable, and the polarization of the calibrator can be ignored during the gain solution. Fomalont \& Perley (1989) provide a good description of the calibration of the Very Large Array (VLA - an alt-az circular configuration). Observations of the phase calibrator are used to first deduce gains, and then to determine the leakages. The gains are a function of time, whereas the leakages are assumed constant. In this process, the phase difference $\psi$ is constrained to be zero and a leakage constraint is added. Because of parallactic angle rotation, calibrator linear polarization can also be deduced for a long synthesis. A final step is to determine the phase difference by observing a strongly linearly polarized source. This overall procedure does not try to fully determine the leakages.

With a parallel-linear configuration the gains and linear polarization cannot be decoupled - a joint solution process is required. Sault et al. (1991) describe the calibration procedure of the Australia Telescope Compact Array (ATCA - an alt-az parallel-linear configuration). They constrain their solution process so that the leakage in one receptor is zero, and they use a noise-injection approach (see Appendix A) to determine the phase difference. They offer two basic strategies - one for a long and one for a short synthesis. For a long synthesis, they solve for the calibrator linear polarization, the antenna gains and the leakages simultaneously. The gains are assumed to vary with time, whereas the leakages are assumed constant over an observation. The strategy for a short synthesis is to assume that the leakages are already known (e.g. by observing another calibrator whose linear polarization is well known) - the linear polarization of the phase calibrator can then be determined simultaneously with the gain solution. Again, neither of these strategies attempts to fully determine the leakages.

For a number of reasons, polarimetric calibration in VLBI is somewhat more involved (e.g. Cotton 1993; Kemball et al. 1995). However, the fundamental limits discussed in the previous sections are not affected by an array with varying antenna mounts, parallactic angles or even receptor types (the details differ, of course).

\section{Self-calibration issues}

Considering an antenna's Jones matrix as the polarimetric equivalent of an antenna gain in non-polarimetric interferometer (e.g. Eq. (3)) makes the applicability of selfcalibration apparent. Indeed Cotton (1993) and Sault \& Wieringa (1994) report using forms of polarimetric selfcalibration. In principle, a self-calibration procedure could solve for the 4 complex coefficients of each antenna Jones matrix for each integration. In this case, the nominal Jones matrix of the antenna (and any parallactic angle rotation) becomes irrelevant. In practice, of course, some instrumental parameters are assumed constant with time, and so decomposing the Jones matrix into different effects (e.g. Eq. (4)) is useful.

Because a polarimetric observation measures four correlations per baseline, it does measure extra closure quantities. Assuming that leakages are negligible, a simple example is the product of correlations $v_{i j, \mathrm{pp}}, v_{j k, \mathrm{pq}}$ and $v_{k i, \mathrm{qp}}$ forms an extra phase closure quantity (ignoring leakage is, of course, ignoring half the polarimetric problem - closure 
quantities independent of leakage will be more involved than a simple triple product). The extra closure quantities will always involve both $v_{\mathrm{pq}}$ and $v_{\mathrm{qp}}$ correlations, which will have poorer signal-to-noise ratio for circular or parallel-linear configurations for most sources. In this case, the advantages of polarization self-calibration may be more related to including leakage and using a source model involving polarimetric properties rather than the extra closure information.

When the advantage of parallactic angle rotation cannot be used, it is clear that the error mechanisms of Sect. 5 cannot be determined by self-calibration. Expressed in another way, the seven degrees of freedom inherent in a pure Mueller matrix cannot be determined by self-calibration. In optical terms, the Mueller matrix of a pure system is not constrained at all if we only know the polarization state of the output (not input).

As a Mueller matrix is concerned only with the polarimetric properties of an optical system, there are other degrees of freedom, in addition to the above seven, that self-calibration cannot determine. For example, absolute position gives two more degrees of freedom, which are not part of a Mueller matrix, that self-calibration cannot determine.

As redundancy and self-calibration share the same fundamental limits in this respect, polarimetric redundancy calibration is also not capable of determining these seven degrees of freedom. Although Noordam (1991) attempted to perform polarimetric redundancy calibration, it is unclear what constraints he added.

There are sometimes astrophysical constraints that can be useful to help eliminate some of these seven degrees of freedom. For example, for a parallel-linear configuration, de Bruyn (1995) has found, for observations containing strong $U$, that minimizing $V$ (when it is known to be astrophysically unlikely) is a useful technique for determining $\psi$. Another possible astrophysical constraint is that the position angle of linear polarization of the source should be in some particular direction.

Because of parallactic angle rotation, the number of unsolvable degrees of freedom in self-calibration is reduced for long observations with alt-az antennas (assuming stable instrumental parameters). We can again analyze the form of Eq. (43) to show that for a source with significant linear polarization, self-calibration can potentially determine four more of the seven degrees of freedom for alt-az antennas. If there is no significant linear polarization, although the four extra degrees of freedom cannot be determined, they cause no corruption in the $I$ and $V$ images. The remaining three degrees of freedom that can never be determined by self-calibration are the absolute flux level, the absolute alignment of linear polarization, and the term which causes $I$ to corrupt $V$ (and vice versa). These must be calibrated externally.
This paper has ignored off-axis polarization of an antenna even though this is a significant effect for some arrays. If the off-axis response is the same for all antennas in an array, and there is no parallactic angle rotation, then the polarimetric errors can be corrected in the image plane. This is the polarimetric equivalent of primary beam attenuation and, similarly, the correction can be delayed until the final analysis stage. This is not the situation when there is parallactic angle rotation, as the off-axis polarization will be far from circularly symmetric. In this case, the resultant polarimetric errors cannot be handled in a straightforward way (see Holdaway et al. 1992 for some approaches), and the usefulness of polarimetric selfcalibration may be significantly limited for large sources.

\section{Conclusions}

A natural question to ask is what are the relative merits of various polarimetric designs, particularly if we can ignore engineering and economic considerations. Clearly an altaz mount (or any mount where the sky and feed assembly rotate relative to each other) has a number of advantages for a long synthesis. For wide-field imaging, however, these advantages may need to be balanced against the problems introduced by the off-axis polarimetric response rotating on the sky.

The question: "Is one receptor configuration better than another?" is somewhat more difficult to answer. If anything, this paper has shown the commonality in the fundamental limits of different receptor configurations. However, receptors do differ in practice. For example, reasonable assumptions allowed Weiler (1973) to almost completely calibrate the crossed-linear configuration by simply observing an unpolarized calibrator. In contrast, for the parallel-linear or circular configurations, a linearly polarized calibrator is needed to determine the phase difference $\psi$ (although a noise-injection system can be used to derive a good estimate of $\psi$ ).

In cases where gain instability is the largest uncorrected error in an observation, it is worth noting that for the circular configuration, gain instability causes $I$ to corrupt $V$, whereas for the parallel-linear configuration, depending on the mount type and alignment of the receptors, $I$ will corrupt $Q$ and/or $U$. So to measure high-accuracy linear or circular polarization, a circular or parallel-linear configuration, respectively, is desirable. In the crossedlinear configuration, gain instability causes $I$ to corrupt all the other parameters, and so from this viewpoint, it is undesirable. However, errors caused by gain instability will be less of an issue when self-calibration is possible.

Although the equations which describe the polarimetric response of circular and parallel-linear configurations are identical apart from a permutation of the Stokes parameters, the sky is not. It is usually a reasonable assumption that circular polarization of many sources is negligible, but the same is often not true for linear polarization. 
This means that a single correlation, $v_{\mathrm{pp}}$ or $v_{\mathrm{qq}}$, is a good measure of $I$ for the circular configuration, but not for linear ones. This results in a number of significant practical advantages for the circular configuration:

- experiments which are not interested in polarimetry need measure only a single correlation.

- gain and leakage calibration solutions can often be decoupled.

- the calibration strategy is often simpler.

Acknowledgements. We thank J.E. Noordam and R.G. Strom for their input and interest in this paper. RJS thanks M.J. Kesteven and N.E.B. Killeen, who stimulated his interest in polarimetry, and with whom the ATCA calibration procedures were developed. The Netherlands Foundation for Research in Astronomy (NFRA) is operated with financial support from the Netherlands Organisation for Scientific Research (NWO). RJS acknowledges support from this organisation under their visiting scientist scheme.

\section{A. Determining the phase difference $\psi$}

As the phase difference $\psi$ is the only large parameter that cannot be determined from an unpolarized calibrator for parallel-linear and circular configurations, we consider some additional techniques to estimate it.

For the Westerbork Synthesis Radio Telescope (WSRT - an equatorial array), which now normally observes in the parallel-linear configuration, a nominal value for the phase difference is determined after each array move by temporarily converting the array to a crossed-linear configuration (the feed assemblies of the antennas are rotatable). This approach was first used by Weiler \& Raimond (1976). For polarimetric observations, observers should determine the error in this nominal value (resulting from, for example, changes with time) by observing a calibrator with strong $U$. The resultant phase difference is then assumed to remain constant during an observation.

The ATCA uses a noise-injection approach - a noise signal is injected (nominally) symmetrically into the p and $\mathrm{q}$ channels in the feed horn. The correlation between the $\mathrm{p}$ and $\mathrm{q}$ channels on a single antenna is then used to derive the phase difference. Receiver-noise arguments imply that this measurement should give $\psi$ to a small fraction of a degree. Current experience suggests that although the measured $\psi$ tracks the true (astronomically determined) $\psi$, because of asymmetries in the noise injection, there is an offset of a degree or so between the two.

A final, rather intriguing, approach is to exploit an offaxis artifact of antennas. For certain antenna/feed designs, an unpolarized source appears polarized, possibly strongly so, when observed off-axis (e.g. Christiansen \& Högbom 1985, page 67; Ghobrial 1976). As this is a characteristic of the mechanical design of the antenna and feed, it is stable and uniform across antennas of the same design. From this artifact, it would be possible to synthesize a polarized source from an unpolarized one - and hence $\psi$ could be determined. Note that this effect cannot be used to determine the absolute alignment or Faraday rotation, as the effect is in the rotation frame of the antennas and not the sky. The accuracy of determining $\psi$ will also probably be limited by the accuracy of the knowledge of off-axis characteristics.

\section{B. Errors in the calibrated Stokes visibilities}

Here we determine the effects of errors in the estimated instrumental parameters. In this appendix, estimates of quantities will be primed, whereas the true values will be unprimed. Using the notation of Paper I, we define the antenna-based receiver matrix as the produce of the gain and leakage matrices,

$$
\boldsymbol{R}_{i}=\boldsymbol{G}_{i} \boldsymbol{D}_{i},
$$

and the baseline-based receiver matrix as

$$
\boldsymbol{R}=\boldsymbol{R}_{i} \otimes \boldsymbol{R}_{j}^{*}
$$

Let us assume that the receiver matrix derived from the calibration procedure is in error by $\Delta \boldsymbol{R}$

$$
\begin{aligned}
\boldsymbol{R}^{\prime} & =\boldsymbol{R}+\boldsymbol{\Delta} \boldsymbol{R} \\
& =\left(\boldsymbol{I}_{4}+\boldsymbol{\Delta} \boldsymbol{R} \boldsymbol{R}^{-1}\right) \boldsymbol{R} .
\end{aligned}
$$

Here $\boldsymbol{I}_{4}$ is the $4 \times 4$ identity matrix. The resultant error in the estimated Stokes vector, $\boldsymbol{\Delta} \boldsymbol{s}$, is

$$
\begin{aligned}
\boldsymbol{\Delta} \boldsymbol{s} & =\boldsymbol{s}^{\prime}-\boldsymbol{s} \\
& =\left(\boldsymbol{K}^{-1}\left(\boldsymbol{I}_{4}+\boldsymbol{\Delta} \boldsymbol{R} \boldsymbol{R}^{-1}\right)^{-1} \boldsymbol{K}-\boldsymbol{I}_{4}\right) \boldsymbol{s} \\
& \approx-\boldsymbol{K}^{-1} \boldsymbol{\Delta} \boldsymbol{R} \boldsymbol{R}^{-1} \boldsymbol{K} \boldsymbol{s} .
\end{aligned}
$$

Here we have assumed that $\boldsymbol{\Delta} \boldsymbol{R}$ is small, and used the approximation

$$
\left(\boldsymbol{I}_{4}+\Delta \boldsymbol{R} \boldsymbol{R}^{-1}\right)^{-1} \approx \boldsymbol{I}_{4}-\Delta \boldsymbol{R} \boldsymbol{R}^{-1} .
$$

To simplify the analysis further, we assume an ideal instrument (gains of 1 and no leakages, i.e. $\boldsymbol{R} \equiv \boldsymbol{I}_{4}$ ). Such an assumption loses no generality; it is in essence simply a renormalization. We are interested in expressing $\boldsymbol{\Delta} \boldsymbol{R}$ in terms of the errors in the gains and leakages. We define these error matrices, $\Delta \boldsymbol{G}_{i}$ and $\boldsymbol{\Delta} \boldsymbol{D}_{i}$, as

$$
\begin{aligned}
\boldsymbol{G}_{i}^{\prime} & =\boldsymbol{I}_{2}+\Delta \boldsymbol{G}_{i} \\
\boldsymbol{D}_{i}^{\prime} & =\boldsymbol{I}_{2}+\boldsymbol{\Delta} \boldsymbol{D}_{i} .
\end{aligned}
$$

If $\Delta g$ is the deviation of a gain from 1 (i.e. the error in the gain), then

$$
\begin{aligned}
\boldsymbol{\Delta} \boldsymbol{G}_{i} & =\left(\begin{array}{cc}
\Delta g_{i \mathrm{p}} & 0 \\
0 & \Delta g_{i \mathrm{q}}
\end{array}\right), \\
\boldsymbol{\Delta} \boldsymbol{D}_{i} & =\left(\begin{array}{cc}
0 & d_{i \mathrm{p}} \\
-d_{i \mathrm{q}} & 0
\end{array}\right) .
\end{aligned}
$$


Because the coefficients of the $\boldsymbol{\Delta}$ matrices are small, we can ignore second-order terms to derive

$$
\Delta R=\Delta G_{i} \otimes I_{2}+I_{2} \otimes \Delta G_{j}^{*}+\Delta D_{i} \otimes I_{2}+I_{2} \otimes \Delta D_{j}^{*} .
$$

Evaluating $\boldsymbol{\Delta} \boldsymbol{s}$ for the parallel-linear case, and using the definitions of Eqs. (36)-(42), results in Eq. (43).

\section{References}

Azzam R.M.A., Bashara N.M., 1987, Ellipsometry and polarized light, North Holland, Amsterdam

Bignell R.C., 1982, in: Thompson A.R., D'Addario L.R. (eds.), Synthesis Mapping: Proceedings of the NRAO-VLA Workshop, Socorro, New Mexico, June 21-25 1982, NRAO, Green Bank, WV

Brouw W.N., 1994 (private communication)

de Bruyn A.G., 1995 (private communication)

Christiansen W.N., Högbom J.A., 1985, Radiotelescopes. Cambridge Univ. Press, Cambridge

Conway R.G., Kronberg P.P., 1969, MNRAS 142, 11

Cotton W.D., 1993, AJ 106, 1241

Fomalont E.B., Perley R.A., 1989, in: Perley R.A., Schwab F.R., Bridle A.H. (eds.), Synthesis Imaging in Radio
Astronomy, ASP, Provo, Utah

Ghorbrial S.I., 1976, IEEE Trans Antenna Propogation, AP24,418

Hamaker J.P., Bregman J.D., Sault R.J., 1996, A\&AS 117, 137

Holdaway M.A., Carilli C.L., Owen F., 1992, VLA Scientific Memo 163, NRAO, Socorro, NM

Kemball A.J., Diamond P.J., Cotton W.D., 1995, A\&AS 110, 383

Kesteven M.J., 1984, AT Document AT/20.1.1/005, ATNF

Morris D., Radhakrishnan V., Seielstad G.A., 1964, ApJ 139, 551

Noordam J.E., 1991, in: Cornwell T.J. and Perley R.A. (eds.), Proc. IAU Col. 131, Radio Interferometry: Theory, Techniques and Applications, ASP, Provo, Utah

Sault R.J., Killeen N.E.B., Kesteven M.J., 1991, AT Tech. Doc. Ser. 39.3/015, ATNF

Sault R.J., Wieringa M.H., 1994, A\&AS 108, 585

Thompson A.R., Moran J.M., Swenson G.W. Jr, 1986, Interferometry and Synthesis in Radio Astronomy. John Wiley $\&$ Sons, New York

Weiler K.W., 1973, A\&A 26, 403

Weiler K.W., Raimond E., 1976, A\&A 52, 397

Weiler K.W., Wilson A.S., 1977, A\&A 58, 17

Weiler K.W., de Pater I., 1983, ApJS 52, 293 